\title{
过渡金属催化的三级胺氧化反应的研究进展
}

\author{
李远明扁娜李志平* \\ (中国人民大学化学系 北京 100872)
}

\begin{abstract}
摘要 三级胺的氧化是自然界中最基本的反应之一. 利用化学方法研究三级胺的氧化反应不仅可以提供合成含氮化合 物的新方法和途径，也可以帮助我们了解生命体中三级胺氧化的反应过程和机理. 近十年, 过渡金属催化的三级胺氧 化反应取得了一些重要的研究进展. 主要总结了近年来以过渡金属为催化剂, 在氧化剂的条件下, 三级胺氧化反应研 究领域中的重要研究结果, 并展望了该研究领域未来研究的重点和挑战性问题. 主要分为五个部分: (1)三级胺的氧化 形成氧化胺; (2)三级胺的氧化 Mannich 反应; (3)三级胺的氧化去甲基化反应; (4)三级胺的氧化酰胺化反应; (5)三级胺取 代基的氧化转化.
\end{abstract}

关键词 三级胺; 氧化; 过渡金属催化反应；去甲基化；酰胺

\section{Progress in the Transition-Metal Catalyzed Oxidation of Tertiary Amines}

\author{
Li, Yuanming Ma, Lina Li, Zhiping* \\ (Department of Chemistry, Renmin University of China, Beijing 100872)
}

\begin{abstract}
The oxidation of tertiary amines is an improtant process in nature. In chemistry, organic chemists have made great efforts to study on the simulation of enzymatic funtion of oxidation with transition metal catalyst. It provide us new and efficient protocols for the synthesis of nitrogen compounds. The catalytic reactions also enhances our understanding the mechanism of the oxidation of tertiary amins in living system. During this decade, significant progress has been made in the transition metal catalyzed oxidation of tertiary amines. This review is summarized by five types of oxidative transformation of tertiary amines: (1) synthesis of $\mathrm{N}$-oxides of tertiary amines; (2) oxidative Mannich reaction of tertiary amines; (3) oxidative demethylation of tertiary amines; (4) oxidative amidation of tertiary amines; (5) oxidative transformation of the substituents of tertiary amines.
\end{abstract}

Keywords tertiary amine; oxidation; transition-metal catalysis; demethylation; amide

三级(叔)胺的氧化是自然界中最基本的反应之一, 在生命过程中起着重要的作用. 例如, 细胞色素 P450催 化的 $N$-去甲基化反应可使化合物的功能激活或者失活, 与生命体新陈代谢中某些生理和病理作用息息相关 ${ }^{[1]}$. 因此, 三级胺的氧化过程引起了生物化学家的极大兴 趣. 由于三级胺是含氮化合物的重要来源, 三级胺的氧 化反应同样受到了有机化学家的广泛关注.

近十年，过渡金属催化的三级胺氧化反应取得了一 系列重要的进展 ${ }^{[2]}$. 根据三级胺的氧化转化途径可以分 为以下五种类型 (Scheme 1): (1)三级胺中氮原子的氧化
(类型 I); (2)氧化 Mannich 反应 (类型 II $)^{[3]}$; (3)三级胺的氧 化去甲基化(类型 III) ${ }^{[4]}$; (4)三级胺的氧化酰胺化反应(类 型 $\mathrm{IV})^{[5]}$; (5)三级胺取代基的氧化转化(类型 $\left.\mathrm{V}\right)^{[6]}$. 本文 将按照以上的反应类型, 对近年来三级胺氧化反应的重 要研究进展进行概述.

\section{1 三级胺的氧化形成氧化胺}

自 1892 年氧化胺第一次被报道 ${ }^{[7]}$ 至今, 氧化胺的化 学已有百年的历史. 氧化胺可以作为氧化剂用于各种氧 化反应, 例如, 锇催化的烯烃的双羟基化 ${ }^{[8]}$, 钓催化的

*E-mail: zhipingli@ruc.edu.cn

Received January 25, 2013; revised March 19, 2013; published online March 22, 2013

Project supported by the National Natural Science Foundation of China (Nos. 21072223, 21272267), the Fundamental Research Funds for the Central Universities, and the Research Funds of Renmin University of China (No. 10XNL017).

国家自然科学基金(Nos. 21072223, 21272267)、中国人民大学科学研究基金(中央高校基本科研业务费专项资金资助)项目成果(No. 10XNL017)资助项 目. 


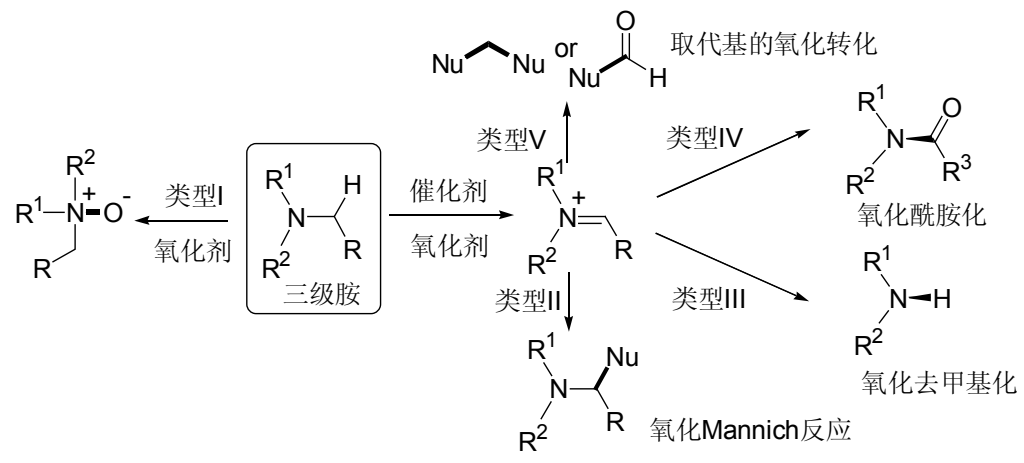

\section{Scheme 1}

醇的氧化 ${ }^{[9]}$, 以及卤代烃转化为醛 ${ }^{[10]}$ 等等. 氧化胺还是 一种多功能弱阳离子表面活性剂, 具有极低的生理毒 性, 广泛应用于餐洗、化妆品、纺织助剂及医药等领 域 ${ }^{[1]}$. 氧化胺也可以作为前驱体, 通过 Cope 消除 ${ }^{[2]}$ 、重 排等 ${ }^{[2 b, 13]}$ 得到一些其它方法难于制备的化合物. 另外, 手性的氧化胺还能作为配体, 实现一些不对称反应 ${ }^{[14]}$.

以双氧水为氧化剂, 通过三级胺的氧化反应是合成 氧化胺最常用的方法 ${ }^{[12,15]}$. 但是, 三级胺氧化反应的选 择性和反应性一直是该研究领域具有挑战性的问题 ${ }^{[16]}$. 研究者们发现过渡金属可以有效地提高三级胺氧化反 应反应速率，并控制氧化反应的选择性 ${ }^{[17]} .2005$ 年, Punniyamurthy 课题组 ${ }^{[17 a]}$ 将钒固载于硅胶制成可重复使 用的催化剂, 与双氧水配合, 可以实现三级胺的绿色、 高效、高选择性的生成氧化胺(Eq. 1).

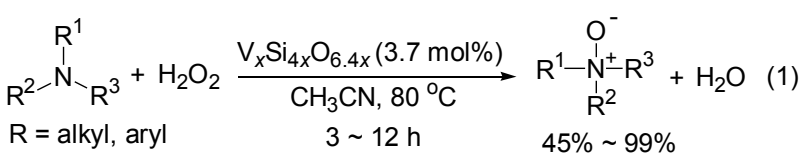

另外, 过酸 ${ }^{[18]} 、 3$-苯基-2-苯基磺酰基-1,2-氧氮杂环 丙烷 ${ }^{[19]}$ 、过氧化酩 ${ }^{[20]}$ 等 ${ }^{[2 b]}$ 也是三级胺氧化反应中的重要 和高效的氧化剂. 氧气毫无疑问是最理想的氧化剂 ${ }^{[21]}$. 2002 年, Sain 课题组 ${ }^{[22]}$ 使用简单的 $\mathrm{RuCl}_{3} \bullet n \mathrm{H}_{2} \mathrm{O} / \mathrm{O}_{2}$ 体系, 以 1,2-二氯乙烷为溶剂, 可以高效的得到氧化胺产物. 该反应的机理一般被认为是, $\mathrm{RuCl}_{3} \bullet n \mathrm{H}_{2} \mathrm{O}$ 和 $\mathrm{O}_{2}$ 能形成 高价钓的氧化物种 ${ }^{[23]}$, 随后氧转移至氮上, 形成氧化胺 (Eq. 2).

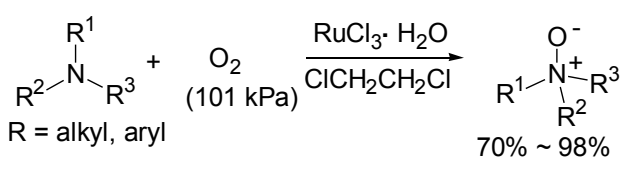

\section{2 三级胺的氧化 Mannich 反应}

Mannich 反应是合成含氮天然产物和活性药物分子 的重要反应之一. 在 Mannich 反应中, 胺与醛首先发生 缩合反应形成亚胺或者亚胺正离子中间体, 进而与亲核
试剂反应得到 Mannich 产物. 形成亚胺或者亚胺正离子 中间体是实现 Mannich 反应的关键步骤. 尽管利用 $\alpha$-取 代基的消除反应也可以得到亚胺正离子，但是通过三级 胺的氧化反应是最直接的一种方法(Scheme 2) ${ }^{[24]}$. 研究 者们成功发展了以钌、铜、铁、钼和钒等过渡金属为催 化剂，以氧气、过氧叔丁醚、过氧叔丁醇等为氧化剂的 催化氧化体系来实现氧化 Mannich 反应. 该部分的研究 进展将以亲核试剂的不同进行分类阐述.

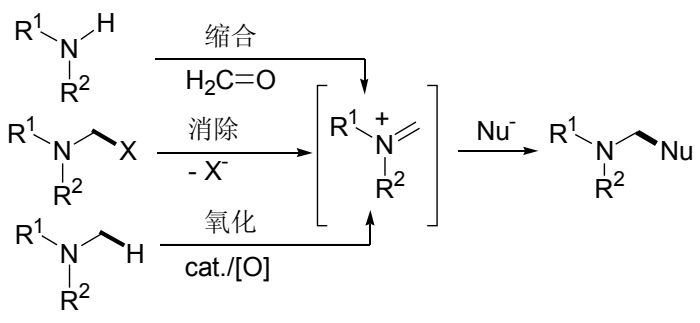

Scheme 2

\section{1 氰化物作为亲核试剂}

2003 年, Murahashi 课题组 ${ }^{[25]}$ 也是利用 $\mathrm{RuCl}_{3} \bullet n \mathrm{H}_{2} \mathrm{O} /$ $\mathrm{O}_{2}$ 体系, 但当这个体系中加入 $\mathrm{NaCN}$ 时, 并不能得到氧 化胺产物, 而是直接实现了芳香三级胺氮邻位的氰基化 (Eq. 3). 亲核试剂的加入, 改变了反应的路径. 该体系 实现了氯基负离子对亚胺正离子的捕捉, 得到 $\mathrm{C}-\mathrm{C}$ 键 的直接偶联的 Mannich 产物. 不足的是, 由于该反应体 系必须使用乙酸，所以在这个体系中会原位产生剧毒的 氢氰酸. 因此, 对氰基源的反应探索也是一个重要方面. 2009 年, Ofial 课题组 ${ }^{[26]}$ 使用 TMSCN 为氧基 源 $^{[27]}$, 利 用 $\mathrm{FeCl}_{2} / \mathrm{TBHP}$ 的氧化体系, 甲醇为溶剂, 既避免了氢氰 酸的产生，也实现了三级胺氮邻位的氰基化. 2012 年, Nageswar 课题组 ${ }^{[28]}$ 采用更加安全、便宜的氰基甲酸乙酯 作为氰基源，也能高效的得到三级胺氰基化的产物.

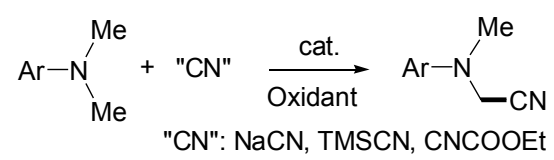




\section{2 富电子芳环作为亲核试剂}

李朝军课题组 ${ }^{[3]}$ 在氧化 Mannich 反应研究领域也做 了一系列创新性的研究工作. 2005 年, 他们 ${ }^{[29]}$ 发现, 在 $\mathrm{CuBr} / \mathrm{TBHP}$ 的体系下, 吲哚的 $\mathrm{C}(3)$ 位能选择性地与四氢 异喹啉底物发生 $\mathrm{C}-\mathrm{C}$ 键氧化偶联反应, 以中等到优秀 的收率得到 Mannich 产物(Eq. 4). 值得注意的是, 当吲 哚 $\mathrm{C} 3$ 位带有取代基时，反应能选择性地得到啊哚 $\mathrm{C}(2)$ 位的偶联产物. 此外, 吲哚苯环上的取代基对该反应的 活性并没有明显的影响. 该反应的局限性在于活性较低 的 $N, N$-二甲基苯胺类衍生物转化率较低 ${ }^{[30]}$. 支志明课题 组 ${ }^{[31]}$ 使用钓卟啉配合物/TBHP 的催化氧化体系, 可以很 好的解决这个问题. 在该课题组进一步的研究中发现, 与钓同一副族的铁与三联吡啶形成的配合物也能高效 催化该类型的反应 ${ }^{[32]}$.

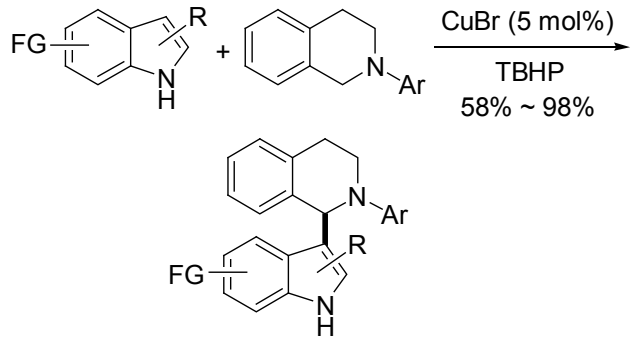

2009 年, Itami 课题组 ${ }^{[33]}$ 使用 $\mathrm{FeCl}_{2} \bullet 4 \mathrm{H}_{2} \mathrm{O}$ 为催化剂, 联吡啶为配体, 吡啶氮氧化物为氧化剂, 实现了富电子 杂芳烃与含甲基三级胺的氧化偶联，以中等收率得到了 目标产物. 值得注意的是, 在该催化氧化体系下, 分子 内氧化偶联反应也能顺利实现. 这在合成氮杂七元环骨 架化合物中具有一定的合成价值和意义(Eq. 5). 但该反 应条件苛刻, 底物范围窄. 2011 年, 张玉红课题组 ${ }^{[34]}$ 发 展的 $\mathrm{CuBr} / \mathrm{O}_{2}$ 体系, 则在温和的条件下高效地实现了吲 嗪类、咪唑类和吲哚等杂环化合物与三级胺的氧化偶联.

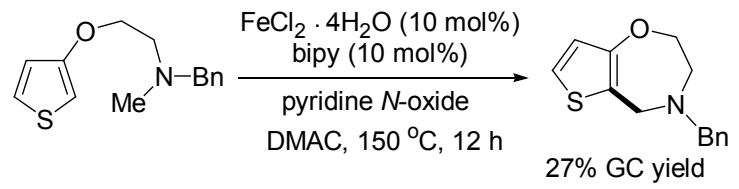

\section{3 炔和烯烃作为亲核试剂}

炔丙胺是合成含氮化合物的重要中间体, 高效地实 现炔丙胺的合成一直是具有挑战性的工作之一. 2004 年, 李朝军课题组 ${ }^{[35]}$ 首次在 $\mathrm{CuBr} / \mathrm{TBHP}$ 体系下, 实现了三 级胺和炔烃的交叉脱氢偶联, 得到炔丙胺类产物(Eq. 6). 该体系只对芳香三级胺和芳香炔烃有较好的适用性. 2008 年, 付华课题组 ${ }^{[36]}$ 利用 $\mathrm{CuBr} / \mathrm{NBS}$ 体系, 解决了活 性低的脂肪三级胺和炔的氧化偶联反应. 2009 年, 在 Vogel 课题组 ${ }^{[37]}$ 发现的 $\mathrm{FeCl}_{2} /(t-\mathrm{BuO})_{2}$ 体系中, 脂肪炔可
以顺利的得到 $\mathrm{C}-\mathrm{C}$ 键的交叉脱氢偶联产物, 并且实现 对位阻小的氮邻位的选择性官能团化.

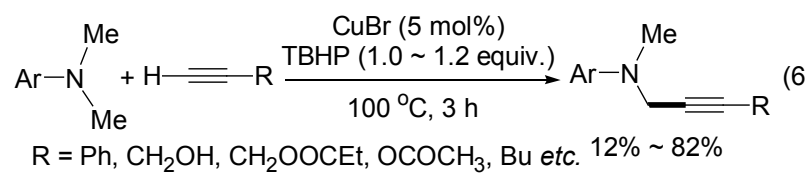

四氢异喹啉 $\mathrm{C}(1)$ 手性碳的合成是天然产物分子以 及药物分子合成中的一个重要反应. 基于 Eq. 6 的反应, 李朝军课题组报道了第一例不对称交叉脱氢偶联(CDC) 反应(Eq. 7 $)^{[38]}$. 当使用 $N$-芳基四氢异喹啉为反应底物, 三氟甲磺酸亚铜为催化剂, 采用具有 C(2)轴对称的手性 噁唑啉为配体时，以中等的对映选择性实现了具有 $\mathrm{C}(1)$ 手性碳的四氢异喹啉衍生物的合成. 王锐课题组 ${ }^{[39]}$ 在以 氧气为氧化剂的体系下，使用三氟甲磺酸铜和有机小分 子协同催化，高对映选择性地实现了四氢异喹啉 $\mathrm{C}(1)$ 位 的不对称烯基化.

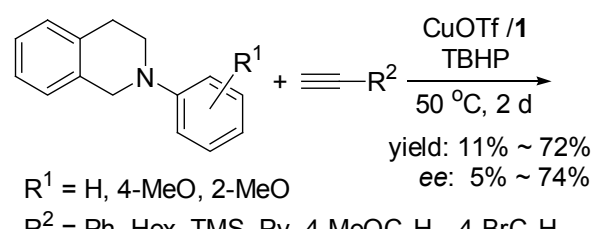

$\mathrm{R}^{2}=\mathrm{Ph}, \mathrm{Hex}, \mathrm{TMS}, \mathrm{Py}, 4-\mathrm{MeOC}_{6} \mathrm{H}_{4}, 4-\mathrm{BrC}_{6} \mathrm{H}_{4}$<smiles>[R]C1=C(c2ccccc2)N(c2ccccc2C)CC1</smiles>

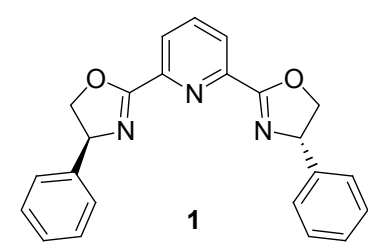

2010 年, Kumaraswamy 课题组 ${ }^{[40]}$ 在 $\mathrm{FeCl}_{3} \cdot 6 \mathrm{H}_{2} \mathrm{O} / \mathrm{T}$ HYDRO 体系中, 首次实现了烯丙基与三级胺的氧化偶 联反应. 当使用手性四氢吡咯做为反应底物时, 可以高 非对映选择性得到氧化偶联产物(Eq. 8).

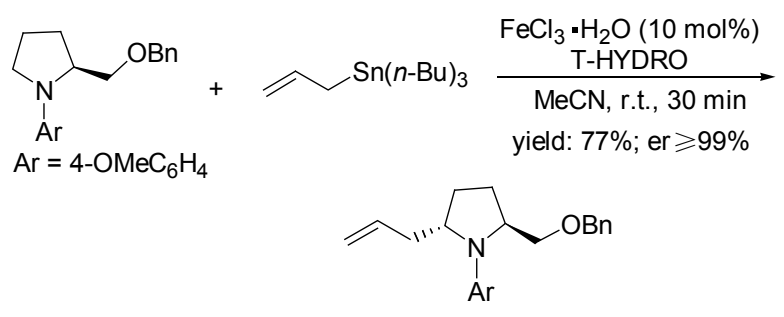

\section{4 烯醇硅醚及酮作为亲核试剂}

含丁内酯骨架的天然产物广泛存在于自然界中, 丁 内酯骨架是天然产物结构中的重要组成部分. 据统计, 
在 $10 \%$ 的已知天然产物中都含有丁内酯骨架. 2004 年, Doyle 课题组 ${ }^{[24]}$ 使用 $\left.\left[\mathrm{Rh}_{2} \text { (cap) }\right)_{4}\right] / \mathrm{TBHP}$ 体系, 以芳香三 级胺和硅烷氧基呋喃为底物, 甲醇为溶剂, 实现了含有 三级胺的 $\gamma$-丁内酯衍生物的合成(Eq. 9). 当采用不对称 三级胺为底物时, 反应选择性的反应在甲基上. 张玉红 课题组 ${ }^{[41]}$ 和卿风领课题组 ${ }^{[2]}$ 分别使用三甲基硅基保护 的酮, 在 $\mathrm{CuBr} / \mathrm{TBHP}$ 体系下, 也能实现对芳香三级胺的 氧化交叉偶联反应.

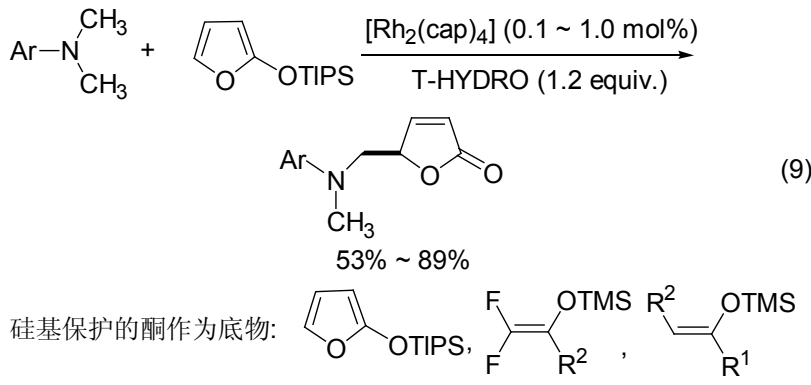

2009 年, 郭灿城课题组 ${ }^{[43]}$ 首次实现了未被保护的 甲基酮与三级胺的氧化交叉偶联反应(Eq. 10). 该研究 采用 $\mathrm{CuI} / \mathrm{O}_{2}$ 体系, 乙酸和分子篮为添加剂. 研究发现, 氧化剂在该体系中具有显著影响. 当采用丙酮作为亲核 试剂时, TBHP 和 $\mathrm{O}_{2}$ 分别为氧化剂时, 可以取得 $63 \%$ 和 $68 \%$ 的收率. 但当采用 2 -丁酮作为亲核试剂时, 加入 TBHP 的反应体系并不能得到偶联产物. 作者认为可能 的原因为: 在氧气的存在下, 体系中可能产生高活性的 铜胺络合物. 2010 年, 黄志真课题组 ${ }^{[44]}$ 采用二级胺活化 酮的策略, 在 $\mathrm{CuBr} / \mathrm{TBHP}$ 体系下, 使三级胺的底物扩展 至 $N, N$-二甲基苯胺的衍生物, 得到了较高收率的目标产 物.

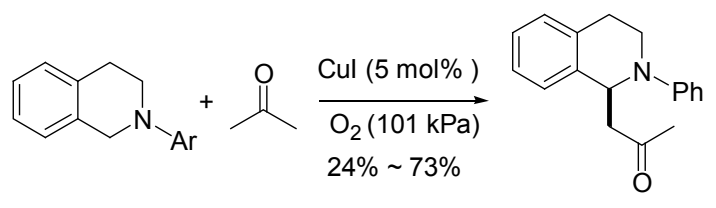

\section{5 含活性氢化合物作为亲核试剂}

2005 年, 李朝军课题组 ${ }^{[3 \mathrm{c}]}$ 实现了三级胺和硝基甲 烷的交叉脱氢偶联反应(Eq. 11). 以 $\mathrm{CuBr} / \mathrm{TBHP}$ 为催化 体系, 在室温条件下, 四氢异喹啉和硝基甲烷偶联得到 一系列 $\beta$-硝基胺衍生物. 这些产物可以方便的还原成 1,2-二胺, 这类物质在药物化学和不对称催化方面具有 重要作用 ${ }^{[45]}$. 当使用 $N, N$-二甲基苯胺为底物时, 由于生 成了去甲基化产物和一些未知的副产物，产率只有 $30 \%$; 但是当使用对位取代的 $N, N$-二甲基苯胺时，反应 效率则大大提高. 其原因可能与三级胺的氧化活性有 关. 2007 年, 李朝军课题组 ${ }^{[46]}$ 发现在 $\mathrm{CuBr} / \mathrm{O}_{2}$ 的条件下,
以水为溶剂, 升高温度至 60 度, 四氢异喹啉与硝基甲烷 反应也能得到优秀的收率. 并且在此条件下，二烷基丙 二酸酯也可以作为良好的亲核试剂与四氢异喹啉反应, 得到 $\mathrm{C}-\mathrm{C}$ 偶联产物.

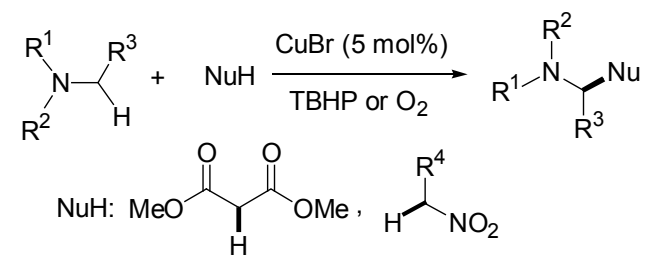

\section{6 杂原子作为亲核试剂}

早在 1988 年, Murahashi 小组 ${ }^{[47]}$ 就报道了在 $\mathrm{RuCl}_{2}\left(\mathrm{PPh}_{3}\right)_{3} / \mathrm{TBHP}$ 体系下, 以苯作为溶剂, 芳香三级胺 与 TBHP 反应高效地得到了过氧化物(Eq. 12). TBHP 在 这个反应不仅是氧化剂，也是亲核试剂。

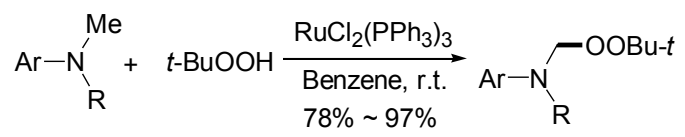

2009 年, 李朝军课题组 ${ }^{[48]}$ 利用 $\mathrm{CuBr} / \mathrm{O}_{2}$ 催化氧化体 系，成功实现了芳香三级胺氮邻位的磷酸化(Eq. 13). 该 反应的不足之处在于反应底物仅局限于 $N$-芳基四氢异 喹啉衍生物. 同年, Ofial 课题组 ${ }^{[49]}$ 利用 $\mathrm{FeCl}_{2}$ /TBHP 催化 氧化体系，以甲醇为溶剂，实现了 $N, N$-二甲基苯胺和亚 磷酸二酯的 $\mathrm{C}-\mathrm{P}$ 氧化交叉偶联反应.

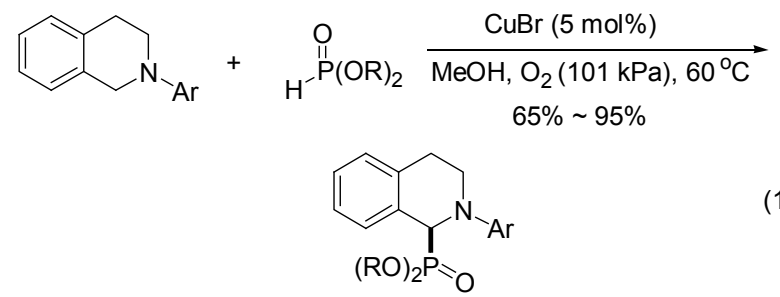

2007 年, 付华和赵玉芬课题组 ${ }^{[50]}$ 使用酰胺作为亲 核试剂，实现了 $\mathrm{C}-\mathrm{N}$ 键的氧化交叉偶联反应(Eq. 14). 研究发现, 在 $\mathrm{CuBr} / \mathrm{TBHP}$ 的催化氧化体系下, 当 $N$-甲 基- $N$-苠基苯胺作为底物时, 脱苯基产物为主要产物.

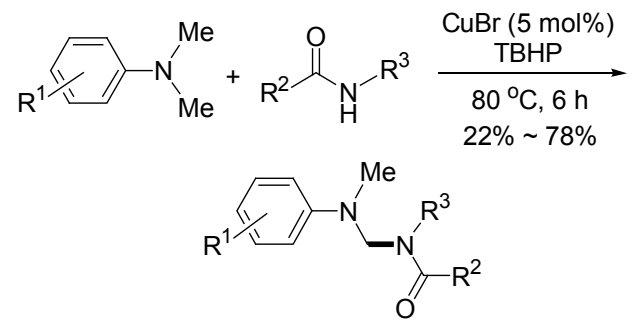

\section{7 机理研究}

氧化 Mannich 反应一般包含两个步骤 ${ }^{[51]}$, 首先, 氧 
化剂在催化剂的作用下, 将三级胺氧化成亚胺正离子; 随后亲核试剂进攻亚胺正离子得到偶联产物. 基于这些 研究结果, 可能的反应机理大致可以归纳为以下通式 (Scheme 3.

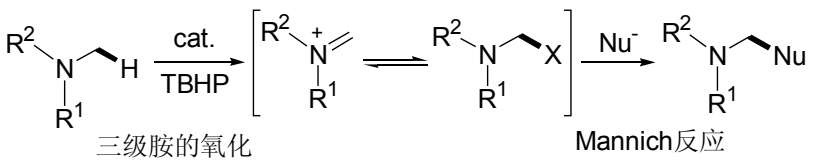

Scheme 3

对于反应中间体的捕捉是机理研究的关键. 多个课 题组通过对中间体的设想和捕捉推测出机理. Doyle 课 题组 ${ }^{[24]}$ 推测在 $\mathrm{Rh}_{2}$ (cap) $_{4} / \mathrm{TBHP}$ 体系中, 当以质子性溶剂 甲醇作为溶剂时, 可以检测到甲醇与亚胺正离子的加成 物. 推测在反应体系中, 甲醇会与硅烷氧基呋喃一起竞 争亚胺正离子(Scheme 4). 支志明课题组 ${ }^{[31]}$ 在 Ru/TBHP 催化的体系中, 以非质子性溶剂甲苯为溶剂时, 则检测 到了过氧叔丁基与亚胺正离子的加成产物, 并且在这个 体系下，该加成产物能转化成产物(Scheme 4). 但是, 并 没有动力学数据可以证明这个过氧化物是中间体. 在 Klussmann 课题组 ${ }^{[52]}$ 的工作中, 以 $\mathrm{CuBr} / \mathrm{TBHP}$ 为催化体 系, 无溶剂反应, 亦得到了过氧化物 $\mathbf{2}$, 经过对反应过程 的检测，可以证明过氧化物 2 是产物的中间体(Scheme
4). 并且，过氧化物 2 可以看作亚胺正离子的“储存库”, 在反应过程中缓慢释放出亚胺正离子，再与硝基甲烷反 应得到最终产物. 在李朝军课题组 ${ }^{[3 \mathrm{c}]}$ 以 $\mathrm{CuBr} / \mathrm{TBHP}$ 为 催化体系中的研究工作中, 则提出假设铜与亚胺正离子 络合形成中间体的假设(Scheme 4).

2013 年, Doyle 课题组 ${ }^{[51]}$ 对以过渡金属为催化剂, TBHP 为氧化剂的 $N, N$ 二 二甲基苯胺的氧化 Mannich 反应 机理做了详细的研究(Scheme 5). Doyle 认为过渡金属 $\left(\mathrm{Rh}_{2} \text { (cap) }\right)_{4}, \mathrm{RuCl}_{3}, \mathrm{CuBr}, \mathrm{FeCl}_{3}$ 等)首先将过氧叔丁醇转 化为过氧叔丁基自由基. $N, N$-二甲基苯胺在过氧叔丁基 的氧化下，经历了一个可逆的单电子转移的过程失去一 个电子. 生成的过氧叔丁醇负离子再将该自由基阳离子 脱去一个质子, 得到 $\alpha$-氨基自由基. 再经过一次的单电 子转移得到亚胺正离子, 随即被具有亲核性质的溶剂或 者是 TBHP 捕捉. 得到的中间体作为亚胺离子的 “储存 库”，来实现亲核试剂与三级胺的氧化偶联. 作者通过 一系列的动力学实验, 证明了这个过程的合理性.

从以上的反应中, 我们可以看到很多催化体系都能 实现三级胺的氧化 Mannich 反应. 在这些催化体系中, 包含 $\mathrm{Ru}, \mathrm{Rh}, \mathrm{Cu}, \mathrm{Fe}, \mathrm{Mo}$ 等过渡金属催化剂; 氧化剂则 可以使用氧气、过氧叔丁醚、过氧叔丁醇等. 当过氧叔 丁醇作为氧化剂时, 常常能以比较温和的条件得到较高
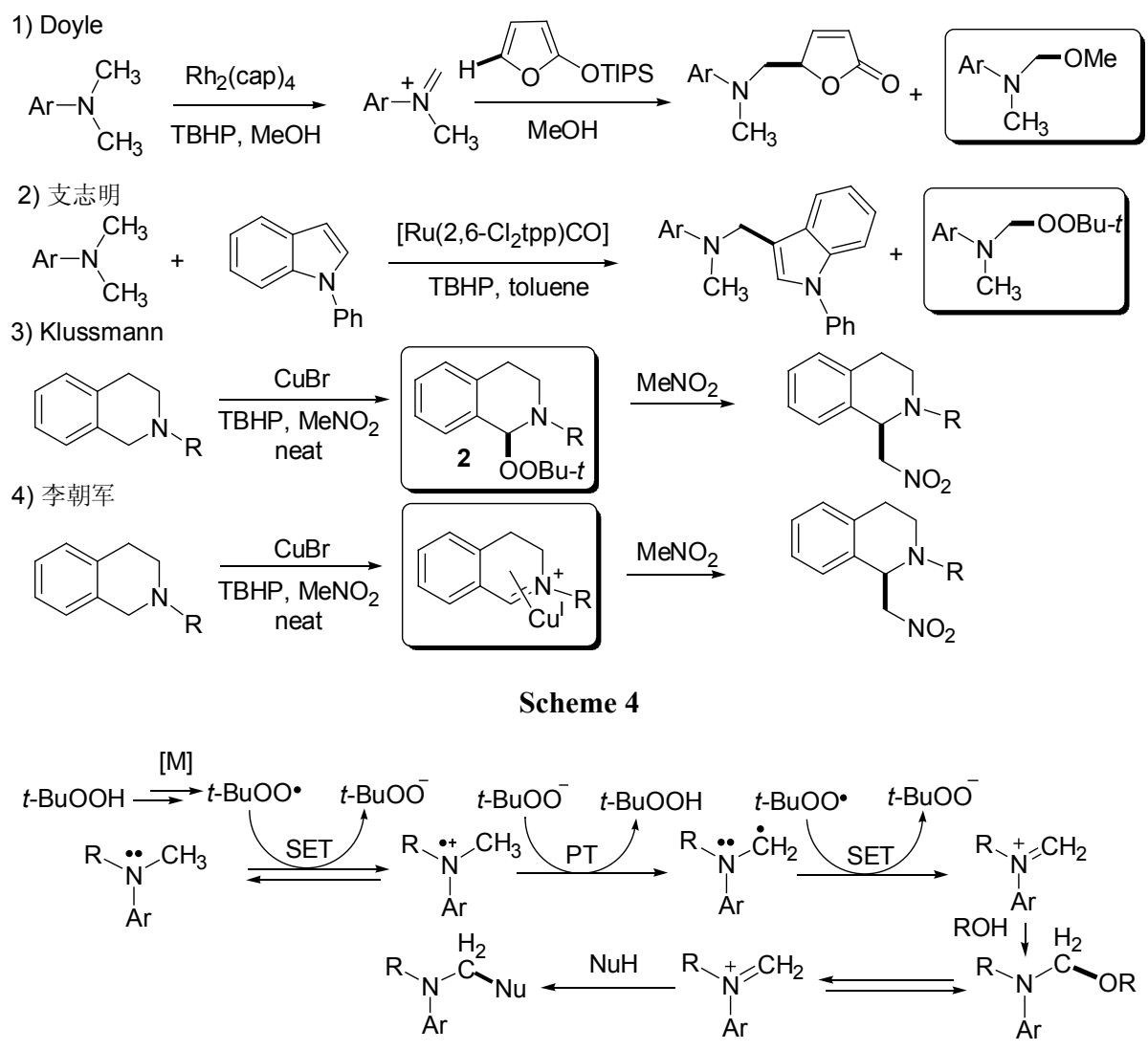

Scheme 5 
产率的目标产物. 使用这些催化体系, 成功的实现了氧 化物、富电子芳环、炔和烯、酮、硝基甲烷、磷酸等亲 核试剂与三级胺的交叉偶联. 其中, 铜盐和过氧叔丁醇 的催化氧化体系适用于大部分亲核试剂与四氢异喹啉 的交叉偶联. 但是当底物为活性较低的 $N, N$-二烷基苯胺 衍生物时, 并没有一个比较通用的体系来实现它与大部 分亲核试剂的交叉偶联. 因此, 寻找一些普适性好、活 性高的催化氧化体系仍然是该研究领域未来发展的重 要研究方向. 最近, 一些新型的普适性好的非金属催化 的氧化体系 ${ }^{[53]}$ 已有报道.

\section{3 三级胺的氧化去甲基化反应}

$N$-甲基三级胺的去甲基化是指通过化学或生物化 学反应从 $N$-甲基三级胺分子中移出甲基基团的过程. 三 级胺的去甲基化反应的研究近年来受到人们广泛的关 注 $^{[54]}$, 其原因在于这类反应与酶催化脱甲基化反应有 关; 并且还可以作为细胞色素 P-450 氧化的模板反应来 进行研究. $\mathrm{N}, \mathrm{N}$-二甲基苯胺的氧化去甲基反应可通过化 学氧化, 电化学氧化 ${ }^{[55]}$ 和光反应 ${ }^{[56]}$ 来实现. 就生物方面 来讲, 由于 DNA 甲基化状态的改变可导致基因结构和 功能异常, 故去甲基化的研究有助于调整基因结构, 使 失去某些生理机能的人恢复正常. 生命新陈代谢过程中 的 $\mathrm{N}$-甲基胺的去甲基化是高效和高选择性的. 但是, 在 化学反应中, 实现高效、高选择性的 $N$-甲基胺的去甲基 化并不容易. 一般要实现三级胺的 $\mathrm{C}-\mathrm{N}$ 键的断裂有两 种策略: (1)通过高价金属氧化物 $(\mathrm{M}=\mathrm{O})$ 的氧化 ${ }^{[57]}$; (2)通 过低价过渡金属的催化氧化 ${ }^{[47,58]}$.

1988 年, Murahashi 课题组 ${ }^{[47]}$ 在 $\mathrm{RuCl}_{2}\left(\mathrm{PPh}_{3}\right)_{3} / \mathrm{TBHP}$ 的催化氧化体系下, 以苯作为溶剂, 三级胺首先与 TBHP 交叉偶联得到过氧化物. 然后, 将过氧化物投入 到盐酸溶液中就能得到去甲基化产物. 该反应的收率较 高. 当底物为不对称三级胺时, 选择性的氧化 $N-\mathrm{CH}_{3}$ (Scheme 6). 1992 年, Murahashi 课题组 ${ }^{[58 c]}$ 以甲醇作为溶 剂时, 在 $\mathrm{RuCl}_{3} \bullet n \mathrm{H}_{2} \mathrm{O} / \mathrm{H}_{2} \mathrm{O}_{2}$ 的体系下, 得到了甲醇与三 级胺的交叉偶联产物. 同样的, 在酸性条件下, 该产物 也能转化为二级胺.

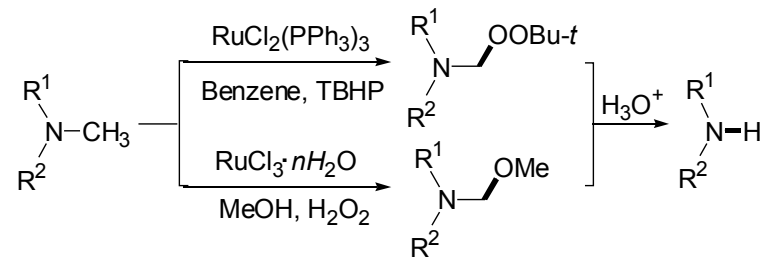

Scheme 6

1989 年, Miura 课题组 ${ }^{[57 e]}$ 发现在铁盐/ $\mathrm{O}_{2}$ 的条件下, 以乙腈为溶剂, $N, N$-二甲基苯胺能被 $\mathrm{O}_{2}$ 氧化为 $N$-甲基苯 胺、 $N$-甲基甲酰苯胺等(Scheme 7). 研究发现, 不同催化 剂对各种反应产物的比例影响较大. 另外, 该课题组还 发现在这个体系中加入 2,6-二叔丁基-4-甲基苯酚(BHT) 时, 当以 $\mathrm{FeCl}_{3}$ 为催化剂时, 对产率影响很小; 然而当采 用 $[\mathrm{Fe}(\mathrm{salen})] \mathrm{OAc}$ 为催化剂时, 反应则完全被抑制. 作 者通过分子内的动力学同位素效应，推测当使用 $\mathrm{FeCl}_{3}$ 为催化剂时, $\mathrm{N}, \mathrm{N}$-二甲基苯胺通过单电子转移得到自由 基阳离子 3, 再失去一个质子, 得到 $\alpha$-氨基自由基 4 (路 径 $\mathrm{a}$ 和 $\mathrm{c}$ ). 而采用 $[\mathrm{Fe}$ (salen)] $\mathrm{OAc}$ 为催化剂时, $N, N$-二甲 基苯胺直接通过氢原子的擢取得到 $\alpha$-氨基自由基 4 (路 径 b). 该反应的缺点是不能得到单一的去甲基化产物. 2011 年, 支志明课题组 ${ }^{\left[{ }^{[7]}\right]}$ 报道了使用铁和低聚吡啶配 合物作为催化剂, 以过硫酸氢钾为氧化剂, 能高选择性 的实现了脂肪和芳香三级胺的去甲基化.

2013 年, 付雪峰课题组 ${ }^{[4]}$ 利用铑卟啉和氧气的体 系, 以水为溶剂, 不仅能实现芳香三级胺的去烷基化, 而且对脂肪三级胺也有很好的适用. 值得注意的是, 当 三级胺为不对称三级胺时, $\mathrm{C}-\mathrm{N}$ 键的断裂更倾向于大 基团的离去(Scheme 8). 该反应的机理大致是三级胺被 (TSPP)Rh(III)氧化, 形成了亚胺正离子的中间体, 催化 剂自身被还原成(TSPP)Rh(I). 亚胺正离子再经过水解得 到二级胺. 最后, 氧气将 (TSPP) Rh(I) 氧化成(TSPP)$\mathrm{Rh}(\mathrm{III})$, 再生催化剂.

\section{4 三级胺的氧化酰胺化反应}

在天然产物、药物和精细化工品的合成过程中, 酰 胺键的形成占有非常重要的地位. 最常见的酰胺的合成 方法是通过活化试剂将羧酸和一级、二级胺缩合得到酰

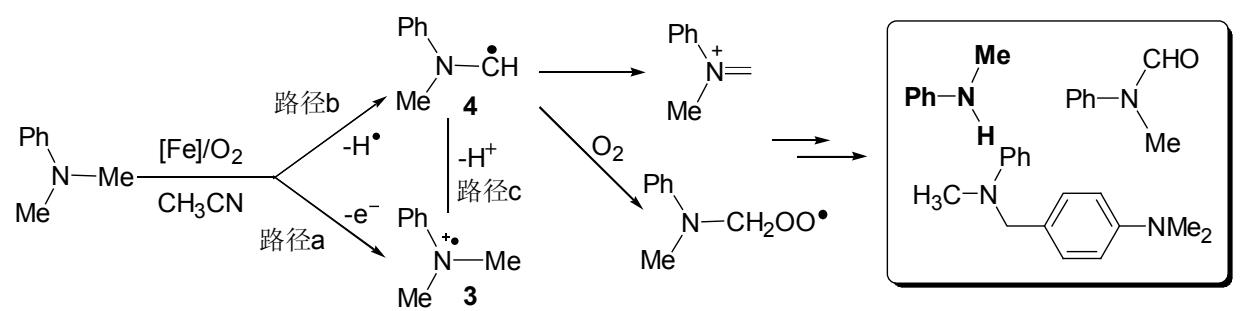

Scheme 7 


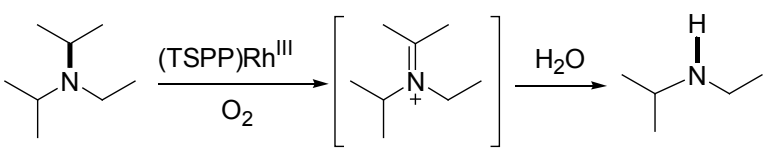

\section{Scheme 8}

胺. 三级胺作为底物来参与酰胺形成的反应目前还是很 少报道. 含 $\mathrm{N}$-甲基的三级胺广泛存在于天然产物之中, 通过 $N$-甲基的去甲基化/酰胺化反应是酰胺键合成中一 个具有挑战性的研究课题. 1927 年, Polonovski 报道了生 物碱的氮氧化物在乙酸酐或乙酰氯的条件下，可以得到 $\mathrm{C}-\mathrm{N}$ 键断裂, 并被乙酰基取代的产物. 随后几十年, 这 个方法都作为三级胺去甲基化的最优方法. 本反应的缺 点是需要先将三级胺氧化成 $N$-氧化物, 分离纯化后再进 行去烷基化酰胺化反应.

1992 年, Miura 课题组 ${ }^{[59]}$ 报道了一步直接将三级胺 转化为去甲基化乙酰化的产物的方法(Eq. 15). 在 5 $\mathrm{mol} \% \mathrm{CoCl}_{2} / \mathrm{O}_{2}$ 的条件下, 以乙腈为溶剂, 4 位取代的 $N, N$-二甲基苯胺和乙酸酐反应，可以生成去甲基酰胺化 的产物和 $N$-烷基苯甲酰胺 $5.0 .2 \mathrm{~mol} \%$ 的 $\mathrm{CoCl}_{2}$ 也能很好 的催化该反应. 并且 $\mathrm{CuCl} / \mathrm{O}_{2}$ 体系也可以催化该反应发 生. 值得注意的是, 虽然有报道 $\mathrm{FeCl}_{3} / \mathrm{O}_{2}$ 体系 ${ }^{[57 \mathrm{e}]}$ 能将 $N, N$-二甲基苯胺去甲基化，但是当采用 $5 \mathrm{~mol} \% \mathrm{FeCl}_{3} / \mathrm{O}_{2}$ 的体系, 却抑制了该反应的发生. 该反应的不足之处是 $N$-烷基苯甲酰胺 5 总是伴随产生, 氧化的化学选择性不 好. 并且酸酕和三级胺的底物范围较窄。作者通过同位 素效应的实验，推测反应的中间体也是亚胺正离子.

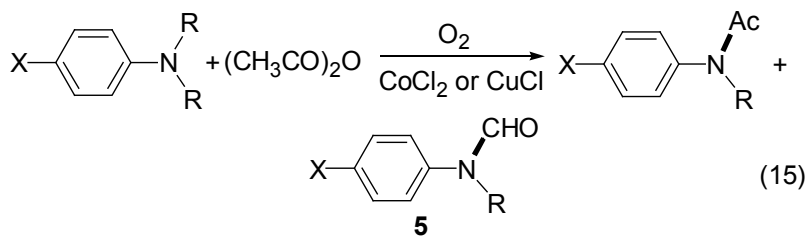

2008 年, Hudlicky 课题组 ${ }^{[60]}$ 报道了 $\mathrm{Pd}(\mathrm{OAc})_{2}$ 催化的 吗啡和莨宕烷类生物碱的去甲基化酰化反应(Eq. 16).

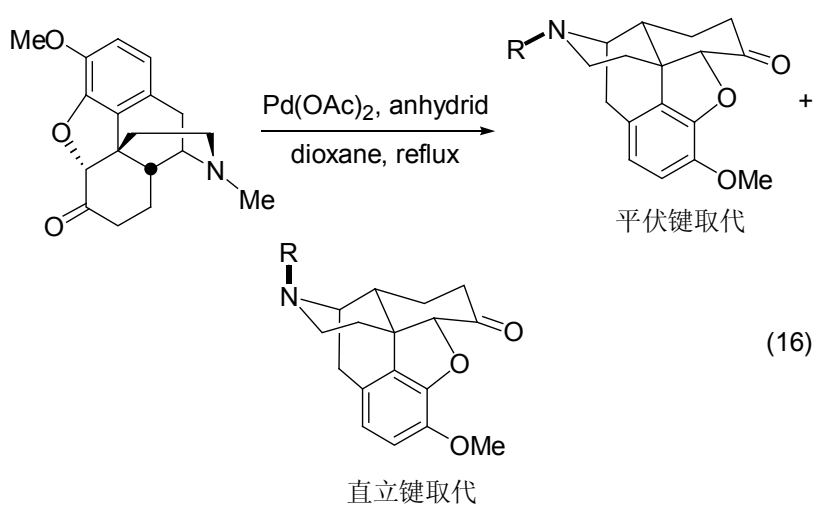

使用 $20 \mathrm{~mol} \%$ 的 $\mathrm{Pd}(\mathrm{OAc})_{2}$ 为催化剂, 以酸酐和二氧六环 为混合溶剂, 可以得到去甲基酰胺化的产物(直立键和 平伏键两种异构体). 不足之处是位阻大的酸酐为底物 时，产物收率低. 托品酮衍生物在这个体系下，也能以 较好的产率得到去甲基酰胺化的产物.

作者提出可能的机理是三级胺被 $\mathrm{Pd}(\mathrm{OAc})_{2}$ 氧化成 亚胺正离子 6, 乙酸根亲核进攻 6 得到加成产物 7; 7 再 经过一系列的反应得到 $\mathbf{8}$, 最终还原消除得到最终产物 (Scheme 9). 在随后的工作中 ${ }^{[61]}$, 该课题组成功将该方 法应用于强效镇痛药丁丙诺啡(Buprenorphine)的合成.

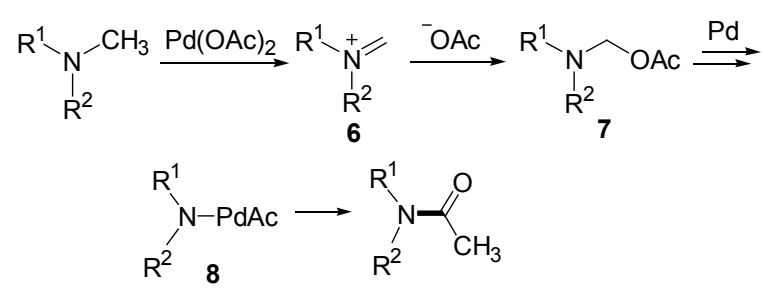

Scheme 9

2013 年, 李志平课题组 ${ }^{[5]}$ 在 $\mathrm{FeCl}_{2} / \mathrm{TBHP}$ 体系下, 首 次实现了醛和三级胺的去甲基酰胺化反应(Eq. 17). 通 过采用 $N, N$-二甲基苯胺和丁醛作为模板底物, 经过一系 列的条件笁选，发现在 $10 \mathrm{~mol} \% \mathrm{FeCl}_{2}$ 的催化下，以 3 equiv. 的 TBHP 为氧化剂, 5 equiv.丁醛为捕捉剂, 乙腈为 溶剂, $85{ }^{\circ} \mathrm{C}$ 反应 $1 \mathrm{~h}$ 可以得到 $90 \%$ 的去甲基丁酰化产物. 醛和三级胺的普适性较好, 三级胺可以是 $N, N$-二甲基苯 胺的衍生物, 也可以是脂肪的三级胺; 脂肪醛和芳香醛 作为底物, 也能高效的转化为产物.

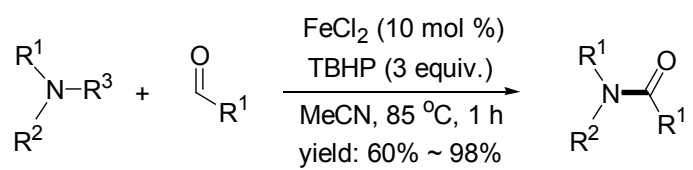

当采用不对称三级胺为底物时，氧化的选择性不 佳. 但是，反应更倾向于去甲基和去苄基(Eq. 18).
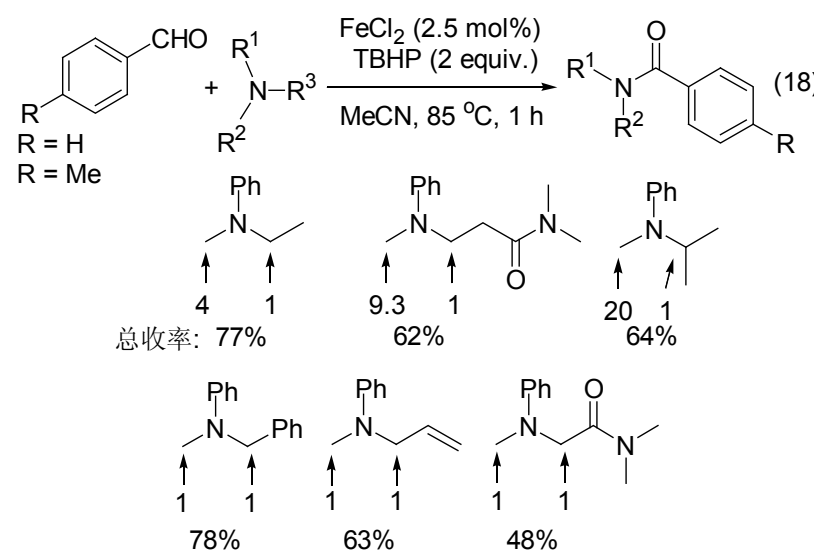
通过 ${ }^{1} \mathrm{H}$ NMR 对反应过程的检测和一系列的对照实 验, 我们推测可能的反应机理(Scheme 10): 首先, 在 $\mathrm{FeCl}_{2} / \mathrm{TBHP}$ 的共同作用下, 形成 $\alpha$-氨基自由基 $\mathbf{9}, \mathbf{9}$ 和过 氧叔丁基自由基可能由于稳态自由基效应(persistent radical effect $)^{[62]}$ 而结合成过氧化合物 10.10 可以分离得 到, 并且在反应过程的核磁检测中, 可以发现 10 在反应 过程中都能保持一定的浓度. 因此我们得出 10 可能是 反应过程的中间体. 10 可以可逆的形成亚胺正离子和过 氧叔丁基负离子, 亚胺正离子再被反应过程中生成的水 捕捉而得到醛和二级胺(该三级胺的去烷基化反应也可 以用于 $N$-芳基化反应 ${ }^{[63]}$ ). 生成的微量二级胺(在 ${ }^{1} \mathrm{H}$ NMR 中未被检测到)迅速与大大过量的醛形成化合物 11, 最后在铁的催化下被氧化成酰胺. 本反应的关键就 是三级胺需要缓慢释放出二级胺. 因此, 当在标准条件 下，用 $N$-甲基苯胺与丁醛反应只能得到 $26 \%$ 的产率; 而 在相同条件下, 采用注射百将 $N$-甲基苯胺在 $1 \mathrm{~h}$ 内注射 入体系, 产率能提高至 $71 \%$.

\section{5 三级胺取代基的氧化转化}

2009 年, 李志平课题组 ${ }^{[6 b]}$ 在 $\mathrm{Fe}(\mathrm{CO})_{9} / \mathrm{TBHP}$ 的体系
下，研究杂原子邻位 $\mathrm{C}-\mathrm{H}$ 键氧化活化中发现，1 equiv. 苯甲酰基乙酸乙酯和 10 equiv. $N, N$-二甲基苯胺作为底 物，可以得到 $47 \%$ 的交叉偶联产物，同时还有 $37 \%$ 的亚 甲基桥联的双 1,3-二羰基化合物 12 (Eq. 19). 将 $N, N$-二 甲基苯胺的量降到 1 equiv.时，反应体系只生成了桥联 的产物. 研究发现, 芳香环上带有吸电子基团的 $N, N$-二 甲基苯胺为底物时，反应产率降低 ${ }^{[57 \mathrm{~d}]}$. 含 $N$-甲基的脂 肪三级胺为底物时, 也具有一定的收率.

可能的反应机理为(Scheme 11): 13 和 14 在铁催化 的氧化体系下先生成氧化偶联的 Mannich 产物 15; 然 后，二羰基化合物 13 对 15 亲核取代得到产物; 或者是 15 经过 Cope 消除得到中间体 16, 再发生 Michael 加成 得到最终产物. 当然, 我们也不能排除二羰基化物与 $N, N$-二甲基苯胺氧化产生的甲醛发生反应得到最终产 物的可能.

在进一步研究其它三级胺的氧化反应中，依然在 $\mathrm{Fe}(\mathrm{CO})_{9} / \mathrm{TBHP}$ 的体系下. 李志平课题组发现, 当使用三 乙胺和 $\beta$-羰基乙酸酯作为反应底物时, 反应得到了 $\beta$-取 代的醛 17 (Eq. 20) ${ }^{[64]}$.

$$
\text { 1 equiv. }
$$
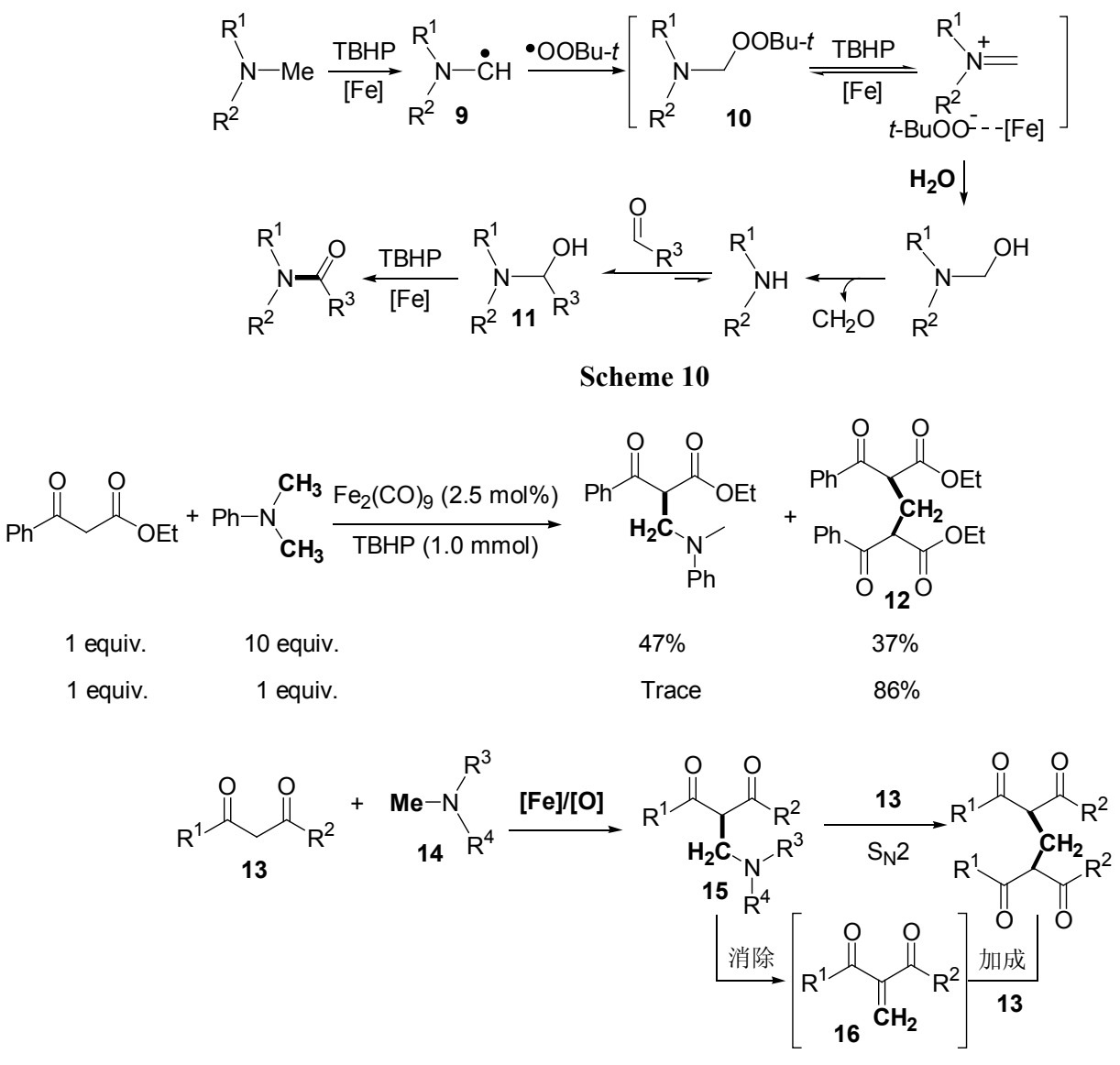

Scheme 11 


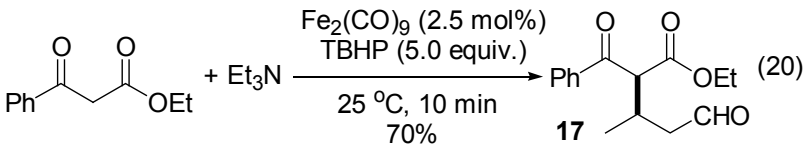

为了探索反应机理, 李志平课题组 ${ }^{[64]}$ 将苯甲酰乙酸 乙酯、乙醛和二乙胺作为底物, 在无氧化剂的条件下, 反应体系中得到了 $69 \%$ 的标准产物(Scheme 12).

结合之前的研究成果, 作者推测在铁催化氧化体系 下，三乙胺首先被氧化成亚胺正离子，亚胺正离子经过 水解得到乙醛和二甲胺. 接下来有两种途径(Scheme 13): (1)路径 a: 两分子乙醛通过差醛缩合得到 21, 苯甲 酰乙酸乙酯 20 进攻 21 得到 Michael 加成的产物. (2)路 径 b: 22 和 23 通过缩合，再发生 Michael 加成得到最终 产物.

2012 年, 李建新课题组在 $\mathrm{CuCl}_{2} / \mathrm{O}_{2}$ 体系 ${ }^{[6 \mathrm{a}]}$ 下，也成 功的使用脂肪的三级胺一一四甲基乙二胺(TMEDA)作

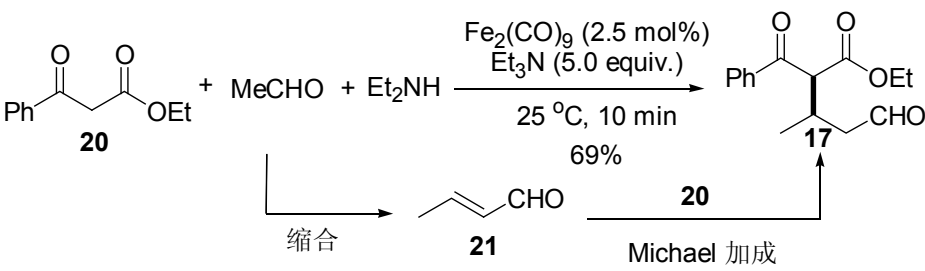

Scheme 12

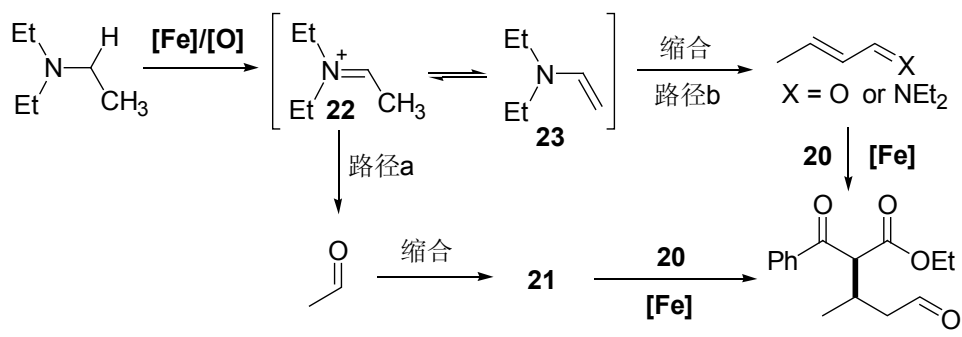

Scheme 13

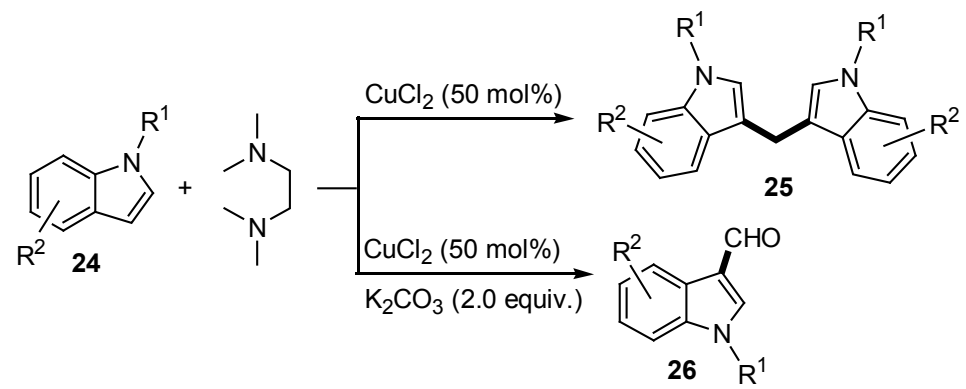

Scheme 14

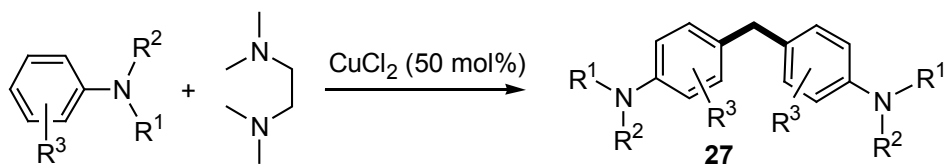




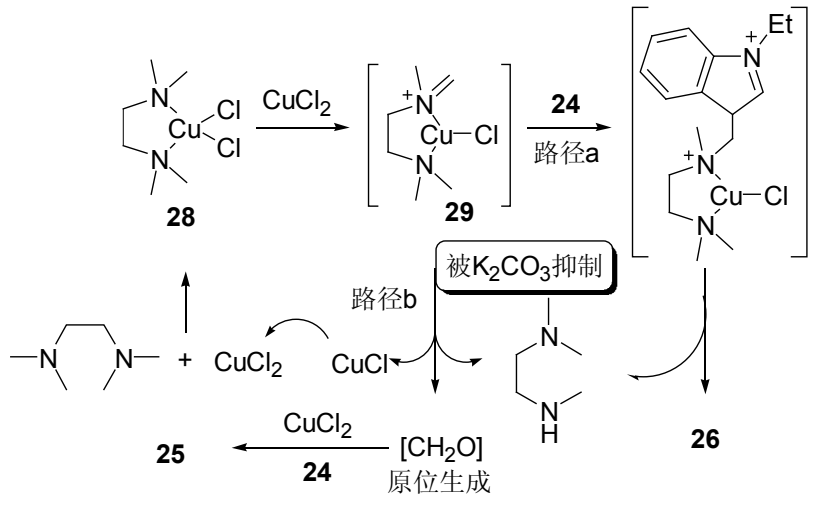

Scheme 15

\section{6 总结与展望}

本文总结了近年来过渡金属催化三级胺氧化反应 的一些重要研究结果和进展. 通过有机化学家们的不断 努力, 三级胺的氧化研究得到了迅猛发展, 不仅反应的 类型越来越丰富, 而且对机理的研究也越来越深入. 亚 胺正离子作为一个关键的中间体, 被催化体系控制着其 随后反应的途径. 在催化氧化体系的发展过程中, 以 钉、铜、铁等金属为催化剂, 以过氧叔丁醇、过氧叔丁 醚、氧气等为氧化剂的催化氧化体系得到长足的发展. 但是, 在三级胺的氧化研究中仍然存在一些挑战性的问 题. 首先, 已有的大多数催化剂只能够用于简单三级胺 的氧化反应, 应用范围较窄. 因此, 寻找普适性好、环境 友好、活性高的催化剂仍然是该研究领域未来发展的重 要研究内容和方向. 其次, 尽管氧气可以用于一些三级 胺的氧化反应, 但是, 大多数反应的选择性不好, 所以 如何有效提高氧气作为氧化剂在反应中的选择性是一 项更具挑战性的研究课题. 另外, 探索三级胺氧化反应 的新途径以及反应机理也同样是该研究领域中有意义 和有挑战性的研究内容.

\section{References}

[1] (a) Meunier, B.; de Visser, S. P.; Shaik, S. Chem. Rev. 2004, 104, 3947.

(b) Ortiz de Montellano, P. R. Cytochrome P-450, Structure, Mechanism, and Biochemistry, 2nd ed., Plenum Press, New York, 1995.

(c) Dawson, J. H.; Sono, M. Chem. Rev. 1987, 87, 1255.

[2] (a) Scheuermann, C. J. Chem. Asian J. 2010, 5, 436.

(b) Bernier, D.; Wefelscheid, U. K.; Woodward, S. Org. Prep. Proced. Int. 2009, 41, 173.

(c) Murahashi, S.-I.; Zhang, D. Chem. Soc. Rev. 2008, 37, 1490.

(d) Murahashi, S.-I. Angew. Chem., Int. Ed. 1995, 34, 2443.

(e) Li, C.-J.; Li, Z. Pure Appl. Chem. 2006, 78, 935.

[3] (a) Guo, X. W.; Li, Z.; Li, C.-J. Prog. Chem. 2010, 22, 1434.

(b) Li, C.-J. Acc. Chem. Res. 2009, 42, 335.

(c) Li, Z.; Li, C.-J. J. Am. Chem. Soc. 2005, 127, 3672.

[4] Ling, Z.; Yun, L.; Liu, L.; Wu, B.; Fu, X. Chem. Commun. 2013,
DOI: $10.1039 / \mathrm{c} 2 \mathrm{cc} 37263 \mathrm{k}$.

[5] Li, Y.; Jia, F.; Li, Z. Chem. Eur. J. 2013, 19, 82.

[6] (a) Zhang, L.; Peng, C.; Zhao, D.; Wang, Y.; Fu, H.-J.; Shen, Q.; Li, J.-X. Chem. Commun. 2012, 48, 5928.

(b) Li, H. J.; He, Z. H.; Guo, X. W.; Li, W. J.; Zhao, X. H.; Li, Z. Org. Lett. 2009, 11, 4176.

[7] Pinner, A.; Wolffenstein, R. Ber. Dtsch. Chem. Ges. 1892, 25, 1428.

[8] Schroeder, M. Chem. Rev. 1980, 80, 187.

[9] Griffith, W. P.; Ley, S. V.; Whitcombe, G. P.; White, A. D. J. Chem. Soc., Chem. Commun. 1987, 1625.

[10] Godfrey, A. G.; Ganem, B. Tetrahedron Lett. 1990, 31, 4825.

[11] Ping, L.; Li, L.; Ping, L. Deterg. Cosmet. 2005, 28, 19 (in Chinese). (李萍, 李丽, 杨旭, 日用化学品科学, 2005, 28, 19.)

[12] Cope, A. C.; Ciganek, E. Org. Synth. 1963, 4, 612.

[13] Albini, A. Synthesis 1993, 263.

[14] Malkov, A. V.; Kočovský, P. Eur. J. Org. Chem. 2007, 29.

[15] VanRheenen, V.; Cha, D. Y.; Hartley, W. M. Org. Synth. 1988, 6, 342.

[16] Wenkert, D.; Angell, E. C.; Chen, T.-F.; Ramachandran, K. Synth. Commun. 1990, 20, 447.

[17] (a) Rout, L.; Punniyamurthy, T. Adv. Synth. Catal. 2005, 347, 1958. (b) Choudary, B. M.; Bharathi, B.; Reddy, C. V.; Kantam, M. L.; Raghavan, K. V. Chem. Commun. 2001, 1736.

[18] (a) Kluge, R.; Schulz, M.; Liebsch, S. Tetrahedron 1996, 52, 5773. (b) Mosher, H. S.; Turner, L.; Carlsmith, A. Org. Synth. 1963, 4, 828.

[19] Zajac, W. W.; Walters, T. R.; Darcy, M. G. J. Org. Chem. 1988, 53, 5856.

[20] Ferrer, M.; Sánchez-Baeza, F.; Messeguer, A. Tetrahedron 1997, $53,15877$.

[21] Jain, S. L.; Sain, B. Angew. Chem., Int. Ed. 2003, 42, 1265.

[22] Jain, S. L.; Sain, B. Chem. Commun. 2002, 1040.

[23] Naota, T.; Takaya, H.; Murahashi, S.-I. Chem. Rev. 1998, 98, 2599.

[24] Catino, A. J.; Nichols, J. M.; Nettles, B. J.; Doyle, M. P. J. Am. Chem. Soc. 2006, 128, 5648.

[25] (a) Murahashi, S.-I.; Nakae, T.; Terai, H.; Komiya, N. J. Am. Chem. Soc. 2008, 130, 11005 .

(b) Murahashi, S.-I.; Komiya, N.; Terai, H. Angew. Chem., Int. Ed. 2005, 44, 6931.

(c) Murahashi, S.-I.; Komiya, N.; Terai, H.; Nakae, T. J. Am. Chem. Soc. 2003, 125, 15312 .

[26] Han, W.; Ofial, A. R. Chem. Commun. 2009, 5024.

[27] (a) Alagiri, K.; Prabhu, K. R. Org. Biomol. Chem. 2012, 10, 835.

(b) Zhang, Y.; Peng, H.; Zhang, M.; Cheng, Y.; Zhu, C. Chem. Commun. 2011, 47, 2354.

[28] Reddy, K. H. V.; Satish, G.; Reddy, V. P.; Kumar, B. S. P. A.; Nageswar, Y. V. D. RSC Adv. 2012, 2, 11084.

[29] Li, Z.; Li, C.-J. J. Am. Chem. Soc. 2005, 127, 6968.

[30] (a) Baslé, O.; Borduas, N.; Dubois, P.; Chapuzet, J. M.; Chan, T.-H.; Lessard, J.; Li, C.-J. Chem. Eur. J. 2010, 16, 8162.

(b) Dombrowski, G. W.; Dinnocenzo, J. P.; Zielinski, P. A.; Farid, S.; Wosinska, Z. M.; Gould, I. R. J. Org. Chem. 2005, 70, 3791.

[31] Wang, M.-Z.; Zhou, C.-Y.; Wong, M.-K.; Che, C.-M. Chem. Eur. J. 2010, 16, 5723.

[32] Liu, P.; Zhou, C.-Y.; Xiang, S.; Che, C.-M. Chem. Commun. 2010, 46, 2739.

[33] Ohta, M.; Quick, M. P.; Yamaguchi, J.; Wunsch, B.; Itami, K. Chem. Asian J. 2009, 4, 1416.

[34] Huang, L.; Niu, T.; Wu, J.; Zhang, Y. J. Org. Chem. 2011, 76, 1759.

[35] Li, Z.; Li, C.-J. J. Am. Chem. Soc. 2004, 126, 11810. 
[36] Niu, M.; Yin, Z.; Fu, H.; Jiang, Y.; Zhao, Y. J. Org. Chem. 2008, 73, 3961.

[37] Volla, C. M. R.; Vogel, P. Org. Lett. 2009, 11, 1701.

[38] (a) Li, Z.; MacLeod, P. D.; Li, C.-J. Tetrahedron: Asymmetry 2006, 17,590 .

(b) Li, Z.; Li, C.-J. Org. Lett. 2004, 6, 4997.

[39] (a) Zhang, G.; Ma, Y.; Wang, S.; Zhang, Y.; Wang, R. J. Am. Chem. Soc. 2012, 134, 12334.

(b) Zhang, G.; Zhang, Y.; Wang, R. Angew. Chem., Int. Ed. 2011, 50, 10429.

[40] Kumaraswamy, G.; Murthy, A. N.; Pitchaiah, A. J. Org. Chem. 2010, 75, 3916.

[41] Huang, L.; Zhang, X.; Zhang, Y. Org. Lett. 2009, 11, 3730.

[42] Chu, L.; Zhang, X.; Qing, F.-L. Org. Lett. 2009, 11, 2197.

[43] Shen, Y.; Li, M.; Wang, S.; Zhan, T.; Tan, Z.; Guo, C.-C. Chem. Commun. 2009, 953.

[44] Yang, F.; Li, J.; Xie, J.; Huang, Z.-Z. Org. Lett. 2010, 12, 5214.

[45] Lucet, D.; Le Gall, T.; Mioskowski, C. Angew. Chem., Int. Ed. 1998, 37, 2580.

[46] Basle, O.; Li, C.-J. Green Chem. 2007, 9, 1047.

[47] Murahashi, S.; Naota, T.; Yonemura, K. J. Am. Chem. Soc. 1988, $110,8256$.

[48] Basle, O.; Li, C.-J. Chem. Commun. 2009, 4124.

[49] Han, W.; Ofial, A. R. Chem. Commun. 2009, 6023.

[50] Zhang, Y.; Fu, H.; Jiang, Y.; Zhao, Y. Org. Lett. 2007, 9, 3813.

[51] Ratnikov, M. O.; Doyle, M. P. J. Am. Chem. Soc. 2013, 135, 1549.

[52] (a) Boess, E.; Schmitz, C.; Klussmann, M. J. Am. Chem. Soc. 2012, 134, 5317.

(b) Boess, E.; Sureshkumar, D.; Sud, A.; Wirtz, C.; Farès, C.; Klussmann, M. J. Am. Chem. Soc. 2011, 133, 8106.

[53] (a) Dhineshkumar, J.; Lamani, M.; Alagiri, K.; Prabhu, K. R. Org. Lett. 2013, 15, 1092.

(b) Tanoue, A.; Yoo, W.-J.; Kobayashi, S. Adv. Synth. Catal. 2013, $355,269$.

[54] Zhang, W.; Yang, L.; Wu, L. M.; Liu, Y. C.; Liu, Z. L. J. Lanzhou Univ. (Nat. Sci.) 1998, 34, 120 (in Chinese).

张炜, 杨立, 吴隆民, 刘有成, 刘中立, 兰州大学学报 (自然科学 版), 1998, 34, 120.

[55] Smith, P. J.; Mann, C. K. J. Org. Chem. 1969, 34, 1821.

[56] Cohen, S. G.; Parola, A.; Parsons, G. H. Chem. Rev. 1973, 73, 141.

[57] (a) Liu, P.; Liu, Y.; Wong, E. L.-M.; Xiang, S.; Che, C.-M. Chem.
Sci. 2011, 2, 2187.

(b) Chiavarino, B.; Cipollini, R.; Crestoni, M. E.; Fornarini, S.; Lanucara, F.; Lapi, A. J. Am. Chem. Soc. 2008, 130, 3208.

(c) Dowers, T. S.; Rock, D. A.; Rock, D. A.; Jones, J. P. J. Am. Chem. Soc. 2004, 126, 8868.

(d) Baciocchi, E.; Lanzalunga, O.; Lapi, A.; Manduchi, L. J. Am. Chem. Soc. 1998, 120, 5783.

(e) Murata, S.; Miura, M.; Nomura, M. J. Chem. Soc., Chem. Commun. 1989, 116.

(f) Karasevich, E. I.; Khenkin, A. M.; Shilov, A. E. J. Chem. Soc., Chem. Commun. 1987, 0, 731.

(g) Smith, J. R. L.; Mortimer, D. N. J. Chem. Soc., Chem. Commun. 1985, 64 .

(h) Shannon, P.; Bruice, T. C. J. Am. Chem. Soc. 1981, 103, 4580.

[58] (a) Maiti, D.; Narducci Sarjeant, A. A.; Karlin, K. D. J. Am. Chem. Soc. 2007, 129, 6720.

(b) Mahapatra, S.; Halfen, J. A.; Tolman, W. B. J. Am. Chem. Soc. 1996, 118, 11575.

(c) Murahashi, S.; Naota, T.; Miyaguchi, N.; Nakato, T. Tetrahedron Lett. 1992, 33, 6991.

(d) Murahashi, S.; Watanabe, T. J. Am. Chem. Soc. 1979, 101, 7429.

[59] Murata, S.; Suzuki, K.; Tamatani, A.; Miura, M.; Nomura, M. J. Chem. Soc., Perkin Trans. 1 1992, 1387.

[60] Carroll, R. J.; Leisch, H.; Scocchera, E.; Hudlicky, T.; Cox, D. P. Adv. Synth. Catal. 2008, 350, 2984.

[61] (a) Machara, A.; Werner, L.; Endoma-Arias, M. A.; Cox, D. P.; Hudlicky, T. Adv. Synth. Catal. 2012, 354, 613.

(b) Machara, A.; Cox, D. P.; Hudlicky, T. Adv. Synth. Catal. 2012, 354, 2713.

[62] Studer, A. Chem. Eur. J. 2001, 7, 1159.

[63] (a) Xie, Y.; Qian, B.; Xie, P.; Huang, H. Adv. Synth. Catal. 2013, DOI: $10.1002 /$ adsc.201200944.

(b) Guo, S.; Qian, B.; Xie, Y.; Xia, C.; Huang, H. Org. Lett. 2010, 13,522 .

[64] Liu, W. P.; Liu, J. H.; Ogawa, D.; Nishihara, Y.; Guo, X. W.; Li, Z. Org. Lett. 2011, 13, 6272.

[65] Chen, J.; Liu, B.; Liu, D.; Liu, S.; Cheng, J. Adv. Synth. Catal. 2012, 354, 2438.

[66] Wu, W.; Su, W. J. Am. Chem. Soc. 2011, 133, 11924. 